\title{
Estimación del impacto de la infección por Hepatitis A en zonas urbanas de Colombia 2008. Un análisis a partir de datos secundarios
}

Grupo de Investigación

en Economía de la Salud, Universidad de Cartagena. 2Facultad de Medicina, Universidad Nacional de Colombia.

3Programa Ampliado de Inmunizaciones, Ministerio de la Protección Social de Colombia.

Financiamiento: El presente estudio fue financiado por el Ministerio de la Protección Social de Colombia en convenio con la Universidad Nacional de Colombia.

Recibido el 24 de agosto de 2009, aceptado el 9 de agosto de 2010.

Correspondencia a: Nelson Alvis

Grupo de Investigación en Economía de la Salud Universidad de Cartagena Telefax: 57-56754454 E-mail: nalvis@yahoo.com

\author{
NELSON ALVIS ${ }^{1}$, BIBIANA PÉREZ ${ }^{2}$, JAVIER NARVAEZ ${ }^{2}$, \\ MARTHA VELANDIA ${ }^{3}$, FERNANDO DE LA HOZ ${ }^{2}$
}

\section{Theoretical estimation of the epidemiological impact of hepatitis A infection in Colombia}

Background: Hepatitis A vaccination is justified in areas with an intermediate endemicity of the infection. Aim: To estimate the epidemiological impact of hepatitis A infection in Colombia. Material and Methods: Epidemiological indicators of hepatitis $A$ infection prevalence by age, morbidity by age, and lethality by age were estimated from a literature search. These measures were projected on a hypothetical cohort of children followed from birth until 15 years of age. The number of cases of infection, jaundice, hepatic failure, hospitalizations and deaths were estimated. Results: From birth to adolescence, a cohort of 872923 urban children in Colombia would generate between 312,331 and 598,591 infections, between 13,586 and 25,960 hospitalizations, between 213 and 407 hepatic failures and between 107 and 204 deaths. Conclusions: The impact of hepatitis A infection in Colombia, is important. Introducing hepatitis A vaccination would reduce a substantial number of severe hepatitis A cases.

(Rev Med Chile 2010; 138: 994-999).

Key words: Hepatitis A; Liver failure; Vaccination.
L a hepatitis A es producida por un virus (VHA) del tipo picornavirus del género de los hepatovirus, su reservorio es el ser humano y el hígado su sitio de replicación ${ }^{1}$. Su transmisión se produce por vía fecal-oral, se asocia con el consumo de agua y alimentos contaminados, contacto persona a persona y además se relaciona con el nivel sanitario e higiénico de la población ${ }^{2}$. Las posibilidades de control y eliminación del VHA son reales ya que el reservorio es exclusivamente humano, no se ha descrito la infección crónica y, si bien se han identificado 4 genotipos a lo largo del mundo, sólo se conoce un serotipo, de distribución universal, que está determinado por epítopes en las proteínas de superficie del virus, posiblemente VP1 y VP3, sin conocerse con precisión contra qué estructura se dirigen los anticuerpos neutralizantes ${ }^{3}$. Actualmente existen al menos 4 vacunas de virus inactivados disponibles, con eficacia y características de seguridad comparables. La eficacia contra la enfermedad clínica se ha encontrado en un rango entre 90 y $100 \%$ para diversos estudios ${ }^{4-6}$.

En Estados Unidos de Norteamérica, el CDC recomienda la vacunación de rutina en los niños residentes en los estados clasificados como de alta ocurrencia de la enfermedad ( $>20$ casos/ 100.000 habitantes) y sugiere que se contemple la vacunación en los estados de ocurrencia intermedia (10-20 casos por 100.000 habitantes $)^{4,7}$. La Organización Mundial de la Salud recomienda la vacunación de acuerdo a la endemicidad. Para 
las zonas de endemicidad intermedia, recomienda la vacunación para los grupos de riesgo: niños, adolescentes, ciertos grupos de trabajadores como los de la salud y manipuladores de alimentos, entre otros $^{8}$. Sin embargo, en los países de desarrollo económico bajo y medio la vacuna es poco usada debido a que la falta de datos epidemiológicos y la percepción de que la vacunación es costosa generan barreras políticas y administrativas para la introducción de la vacuna9.

En Colombia, en el 2006 se notificaron 4.212 casos de ictericia ligados a infección reciente por hepatitis A, para una incidencia media estimada de entre 8,0 y 11,5 casos por 100.000 habitantes, respectivamente. El grupo etáreo más afectado en el año 2006 fue el de 5 a 14 años seguido por el de 15-44 años ${ }^{10}$. Sin embargo, como en otros países de América Latina, la información epidemiológica sobre la frecuencia de la infección con hepatitis A y sus consecuencias es parcialmente desconocida, debido a la baja cobertura del sistema de vigilancia sobre este agente y a dificultades para la confirmación por laboratorio de las causas de ictericia. Debido a estas limitaciones, el Ministerio de la Protección Social planteó la necesidad de estimar el impacto global de la infección por el virus de la hepatitis A en Colombia, con el fin de considerar la introducción de la vacunación universal.

\section{Material y Método}

Se realizó una revisión bibliográfica de la literatura latinoamericana sobre epidemiología de la infección por hepatitis A y un análisis descriptivo de algunas fuentes de información rutinaria del Instituto Nacional de Salud, el Ministerio de la Protección Social y algunas Secretarias de Salud del país.

Revisión bibliográfica: Los datos demográficos se tomaron de las proyecciones de población del DANE con base en el censo de $2005^{11}$. Para la revisión bibliográfica de Literatura Latinoamericana se consultaron las bases de datos: MEDLINE (Gateway), LiLacs y Scielo. Los términos Mesh usados fueron: "Not adult", "Hepatitis A", "Prevalence", "Incidence", "hospitalization", "critical illness", "liver failure", "mortality" "America Not North America". Los criterios de inclusión de los estudios fueron los siguientes: estudios con datos originales, en inglés, español o portugués y con fecha de publicación posterior a 1995, con indica- dores epidemiológicos de incidencia, prevalencia, mortalidad, severidad y hospitalizaciones que hubieran sido recolectados en algún país de Latinoamérica y el Caribe. Estos estudios se revisaron en texto completo pero se decidió usar para las estimaciones sólo aquellos de zonas urbanas donde la muestra era de población general y seleccionada aleatoriamente.

Se identificaron inicialmente 60 artículos en Latinoamérica cuyo título estaba referido a hepatitis A (22 en español, 21 en inglés y 17 en portugués). Estos procedían de Brasil $(\mathrm{n}=32)$, Argentina $(\mathrm{n}=6)$, Cuba $(\mathrm{n}=6)$, Chile $(\mathrm{n}=4)$, Costa Rica $(\mathrm{n}=4)$, Perú $(\mathrm{n}=2)$, Uruguay $(\mathrm{n}=$ $2)$, Bolivia $(n=1)$, México $(n=1)$, Nicaragua ( $n$ =1) y Venezuela $(\mathrm{n}=1)$. Los años de publicación estuvieron entre 1997 y 2007 y 57 de los 60 se realizaron en la zona urbana. De estos artículos sólo 24 cumplían con los criterios de inclusión y la mayoría eran estudios transversales $(n=22)$. Sólo 4 mencionaban la forma como se habían obtenido las muestras de población incluidas en el estudio y de estos, 2 fueron seleccionados para la extracción de la información epidemiológica sobre prevalencia de la infección por hepatitis A ya que habían sido realizados en muestras de población general seleccionada aleatoriamente $e^{2,9,12.13}$.

Estimación de parámetros epidemiológicos: $\mathrm{Se}$ hizo a partir de los estudios realizados en Santiago de Chile y Caracas. De ellos se extrajeron los datos de prevalencia por grupos de edad que luego fue desagregada por unidades de edad (1 año) usando un modelo de regresión logarítmica. Posteriormente, se calculó la incidencia (riesgo) de infección por hepatitis A para cada año de edad usando la transformación propuesta por Kleinbaum et al y Leske et $\mathrm{al}^{14,15}$, para estimar incidencia acumulada a partir de datos de prevalencia. El número de nuevos infectados para cada año de vida (casos incidentes/año), estimado a partir de la prevalencia de infección, se multiplico por las probabilidades de desarrollar ictericia o falla hepática o muerte según la Tabla 1. No se encontraron estudios latinoamericanos que mostraran claramente las características clínicas de la infección por hepatitis A como la proporción de casos que desarrollan enfermedad ictérica, que requieren hospitalización, que desarrollan falla hepática, etc. Por ello estos indicadores se extrajeron de la literatura médica universal (Tabla 1). La definición de infección, en los artículos usados para las estimaciones, se basó 
Tabla 1. Prevalencia y probabilidades de infección por Hepatitis A por grupo etáreo

\begin{tabular}{|c|c|c|c|c|}
\hline \multicolumn{5}{|c|}{ Prevalencia de infección por Hepatitis A } \\
\hline Ciudad (Año) & 1 a 4 años & 5 a 9 años & 10 a 15 años & Referencias \\
\hline Caracas (1999) & $27,3 \%$ & $54,1 \%$ & $61,7 \%$ & $\begin{array}{l}\text { (Tapia-Conyer, Santos } \\
\text { et al. 1999) }\end{array}$ \\
\hline Santiago (2002) & $12,5 \%$ & $26,2 \%$ & $43,4 \%$ & $\begin{array}{l}\text { (Fix A. D., Martin et } \\
\text { al. 2002) })^{13}\end{array}$ \\
\hline \multicolumn{5}{|c|}{ Probabilidades de manifestaciones clínicas y enfermedad severa } \\
\hline Ictericia & $7,2 \%(4,7 \%-10,9 \%)$ & $37,1 \%(30,7 \%-43,8 \%)$ & $70,7 \%(58,8 \%-79,4 \%)$ & $\begin{array}{l}\text { (Armstrong G. L. and } \\
\text { Bell 2002) }{ }^{17}\end{array}$ \\
\hline Hospitalizados & $5 \%$ de los ictéricos & $17 \%$ de los ictéricos & $17 \%$ de los ictéricos & $\begin{array}{l}\text { (Rein D. B., Hicks K. } \\
\text { A. et al. 2007) }\end{array}$ \\
\hline Falla hepática & $\begin{array}{l}0,24 \% \text { de los } \\
\text { ictéricos }\end{array}$ & $0,24 \%$ de los ictéricos & $0,24 \%$ de los ictéricos & $\begin{array}{l}\text { (Armstrong G. L. and } \\
\text { Bell 2002) }{ }^{17}\end{array}$ \\
\hline Mueren & $\begin{array}{l}50 \% \text { de las Fallas } \\
\text { Hepáticas }\end{array}$ & $\begin{array}{l}50 \% \text { de las Fallas } \\
\text { Hepáticas }\end{array}$ & $\begin{array}{l}50 \% \text { de las Fallas } \\
\text { Hepáticas }\end{array}$ & $\begin{array}{l}\text { (Armstrong G. L. and } \\
\text { Bell 2002; Rein D. } \\
\text { B., Hicks K. A. et al. } \\
\text { 2007) }\end{array}$ \\
\hline
\end{tabular}

en el criterio serológico de positividad a IgG para hepatitis A.

Estimación del número de infectados y enfermos: Se construyó una cohorte hipotética de niños recién nacidos $(\mathrm{n}=872.923)$ en centros urbanos colombianos que fueron seguidos, desde el nacimiento hasta cumplir los 15 años, el número de personas vivas cada año fue ajustado de acuerdo a las tablas de vida del DANE para 2005. A la cohorte se le aplicó el riesgo de infección por hepatitis A para cada año de edad, estimado partir de la literatura y descritos en el párrafo anterior. Al número de personas infectadas en cada año de edad se les aplicaba las probabilidades de desarrollar ictericia, enfermedad severa y muerte que se muestran en la Tabla 1. De esa manera, para cada año de vida se estimaba el número de infectados, de ictéricos, de fallas hepáticas y de muertes y el número total de casos esperados para la cohorte se obtuvo sumando los estimados anuales.

\section{Resultados}

La Figura 1 muestra el riesgo de infección por el virus de la hepatitis A, asumiendo el comportamiento serológico observado en Santiago de Chile o Caracas. Puede verse como hay un pico de infección en los dos primeros años de vida y luego el riesgo de infección cae a 3\% a los dos años de vida pero luego iría aumentando gradualmente hasta alcanzar el 5\% anual alrededor de los 12 años.

En la Tabla 2 se muestra la frecuencia de enfermedad distribuida por diferentes grados de severidad, que se presentaría en una cohorte hipotética de nacidos vivos en Colombia, seguida por un período de 15 años, asumiendo dos patrones de infección (Santiago de Chile y Caracas) y aplicando las probabilidades de estar infectado, hacer enfermedad ictérica, ser hospitalizado, desarrollar falla hepática y morir, descritas en la literatura y reportados en la Tabla 1.

Como puede observarse el grupo etáreo con mayor frecuencia de infección es el de 0 a 4 años, sin embargo, el que mayor frecuencia de ictericia, hospitalización, falla hepática y muerte presenta es el de 10 a 14. Usando la seroprevalencia observada en Santiago de Chile se espera que a los 15 años alrededor de 470.000 niños hayan sido infectados con el VHA y que estas infecciones hayan generado 176 muertes y 350 fallas hepáticas para el mismo período. Usando los datos de Caracas el número máximo de infectados esperado, después de 15 años, seria aproximadamente de 600.000 los cuales producirían 204 muertes y 407 casos de falla hepática. 


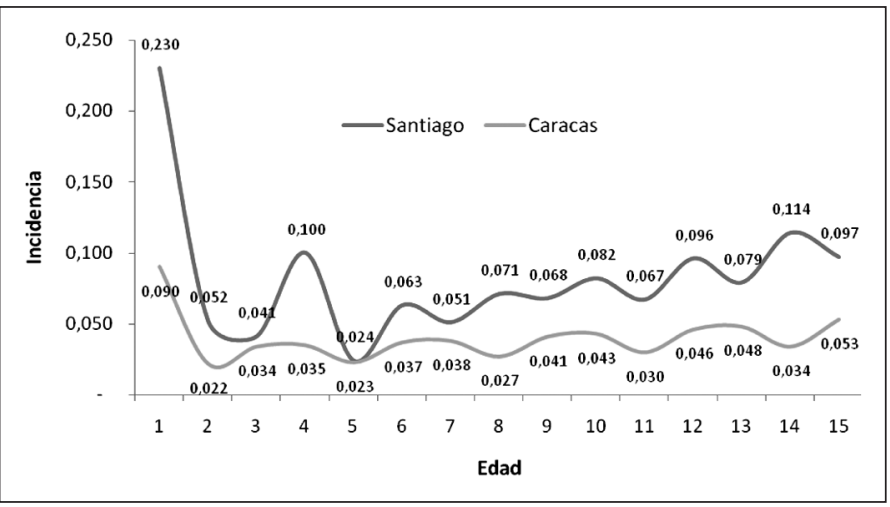

Figura 1. Incidencia por edad de la infección por VHA en Santiago de Chile y Caracas.

Tabla 2. Estimación de casos de Infección por Hepatitis A por grupos de edad y patrones de infección en Colombia

\begin{tabular}{|c|c|c|c|c|c|c|}
\hline Edad & Referencia & $\begin{array}{c}n \text { de } \\
\text { infecciones }\end{array}$ & $\begin{array}{c}\text { n de } \\
\text { ictéricos }\end{array}$ & $\begin{array}{c}\text { n de } \\
\text { hospitalizados }\end{array}$ & $\begin{array}{l}\text { n de falla } \\
\text { hepática }\end{array}$ & $\begin{array}{c}\text { n de } \\
\text { muertes }\end{array}$ \\
\hline \multirow[t]{2}{*}{0 a 4} & Chile & 233.312 & $\begin{array}{c}16.798 \\
(10.966-25.431)\end{array}$ & $\begin{array}{c}840 \\
(548-1.272)\end{array}$ & $\begin{array}{c}40 \\
(26-61)\end{array}$ & $\begin{array}{c}20 \\
(13-31)\end{array}$ \\
\hline & Venezuela & 328.245 & $\begin{array}{c}23.634 \\
(15.428-35.779)\end{array}$ & $\begin{array}{c}1.182 \\
(771-1.789)\end{array}$ & $\begin{array}{c}57 \\
(37-86)\end{array}$ & $\begin{array}{c}29 \\
(19-43)\end{array}$ \\
\hline \multirow[t]{2}{*}{5 a 9} & Chile & 119.704 & $\begin{array}{c}44.410 \\
(36.749-52.430)\end{array}$ & $\begin{array}{c}7.550 \\
(6.247-8.913)\end{array}$ & $\begin{array}{c}107 \\
(87-126)\end{array}$ & $\begin{array}{c}54 \\
(44-63)\end{array}$ \\
\hline & Venezuela & 135.080 & $\begin{array}{c}50.115 \\
(41.470-59.165)\end{array}$ & $\begin{array}{c}8.520 \\
(7.050-10.058)\end{array}$ & $\begin{array}{c}120 \\
(100-142)\end{array}$ & $\begin{array}{c}60 \\
(50-71)\end{array}$ \\
\hline \multirow[t]{2}{*}{10 a 14} & Chile & 119.754 & $\begin{array}{c}84.666 \\
(70.415-95.084)\end{array}$ & $\begin{array}{c}14.393 \\
(11.971-16.164)\end{array}$ & $\begin{array}{c}203 \\
(169-228)\end{array}$ & $\begin{array}{c}102 \\
(85-114)\end{array}$ \\
\hline & Venezuela & 135.266 & $\begin{array}{c}95.633 \\
(79.536-107.401)\end{array}$ & $\begin{array}{c}16.258 \\
(13.521-18.258)\end{array}$ & $\begin{array}{c}230 \\
(191-258)\end{array}$ & $\begin{array}{c}115 \\
(96-129)\end{array}$ \\
\hline
\end{tabular}

\section{Discusión}

La carga de enfermedad por hepatitis A en Colombia, cuando se estima hasta los 15 años, es importante, si tenemos en cuenta que en una cohorte de 872.923 nacidos vivos pueden ocurrir entre 80 y 200 muertes por falla hepática debida a VHA. La vacuna inactivada contra hepatitis A es altamente efectiva, como ha sido demostrado en numerosos estudios y una sola dosis podría proteger a largo plazo en una proporción mayor de $80 \%{ }^{19}$. Por ello, podría ser justificado introducir la vacunación universal en menores de 5 años pues contribuiría a reducir cerca de $5 \%$ de las muertes que suceden entre 5 y 15 años en Colombia DANE ${ }^{20}$.
Varias son las potenciales debilidades que deben considerarse al momento de interpretar los resultados de este estudio. La primera es que al momento de hacer las estimaciones no existían estudios serológicos colombianos sobre muestras representativas de la población. Por ello, se recurrió a datos de otros países de Latinoamérica lo que puede llevar a sobreestimar o subestimar la carga para Colombia. Sin embargo, los datos usados venían de poblaciones urbanas en países con niveles de desarrollo similares a los de Colombia y con similares porcentajes de población urbana que son los factores que más influencian la epidemiologia de la hepatitis $\mathrm{A}^{2,12,21}$. Adicionalmente, los niveles de infección usados en nuestro estudio 
son consistentes con un patrón de endemicidad intermedia que es el perfil epidemiológico de hepatitis A aceptado para Latinoamérica lo que da coherencia a nuestras estimaciones $^{8,22}$.

Otra limitación es que no existen buenos datos de seroprevalencia de hepatitis A para poblaciones rurales y municipios pequeños en Latinoamérica o Colombia que podrían tener un patrón epidemiológico diferente al de las grandes ciudades, debido a mayor precariedad en el acceso a agua potable. Esto podría llevar a que nuestros cálculos de carga de enfermedad tengan cierto nivel de sobreestimación. Sin embargo, menos de 30\% de los niños colombianos viven en tales áreas por lo que la sobre estimación de la carga no sería tan importante.

Otra dificultad es la falta de información contra la cual validar nuestros estimados. Claramente los estimados del SIVIGILA para el país son subestimados si comparamos los datos de la vigilancia provenientes de Bogotá contra los que vienen de otras zonas del país. Menos información aun hay sobre las muertes que puedan ser ocasionadas por el virus de la hepatitis A. Nuestros estimados predicen entre 80 y 200 muertes en personas de 0 a 15 años, y entre 160 y 377 casos de falla hepática para el mismo intervalo de edad pero el número de muertes en ese grupo de edad que pueden ser causados por hepatitis A es bastante menor que ese estimado. Para el 2001 por ejemplo, el DANE reporto 30 muertes en menores de 15 años por todos los síndromes hepáticos que podrían deberse a hepatitis A, es decir menos de la tercera parte del límite inferior de nuestros estimados.

Estudios recientes en Argentina, Chile y Brasil, muestran que hasta el $64 \%$ de los casos de falla hepática fulminante en niños que requieren trasplante de hígado se asocian a infección por VHA $^{23-26}$. En Colombia, se han reportado unos 40 trasplantes de hígado en los últimos dos años en menores de 15 años, si extrapolamos los datos de los otros países del continente entonces el virus de la hepatitis A podría estar causando entre 20 y 30 casos de trasplantes en este grupo de edad por año. Sin embargo, expertos nacionales consultados afirmaron que la causa principal en el país es por cirrosis causada por otros virus o por falla hepática de origen medicamentoso.

El cambio de los patrones de prevalencia de infección por VHA hace más vulnerables a los grupos de adolescentes y adultos en donde el curso de la enfermedad puede tener manifestaciones fatales. A pesar de que actualmente existen vacunas seguras y eficaces para controlar el virus VHA, estas no han sido incluidas en los programas de vacunación masiva de la mayoría de países latinoamericanos, por sus elevados costos.

Por último, se recomienda reforzar la vigilancia de las ictericias fortaleciendo los laboratorios de salud pública y la capacidad para analizar las bases de datos de los prestadores de servicios. Esto permitirá contar, al menos, con un estimado del universo de enfermedades parecida a hepatitis virales, lo cual ayudaría a llenar el vacío de conocimiento alrededor de la magnitud y el impacto de este síndrome. Es imprescindible fomentar la implantación de puestos de vigilancia centinela, donde se haga diagnóstico de todos los cuadros sospechosos de hepatitis viral y poder contar con mejores datos descriptivos que nos permitan identificar, de manera más confiable, la proporción de casos de ictericia que pueden estar asociados a hepatitis A. Adicionalmente, estos mismos centros pueden vigilar la ocurrencia de hepatitis virales que necesitan hospitalización y cuantificar la frecuencia en que están asociados con hepatitis A.

Declaratoria de conflicto de intereses. La doctora Martha Velandia es la coordinadora del Programa Ampliado de Inmunizaciones de Colombia. El resto de autores manifiesta no tener conflicto de intereses.

\section{Referencias}

1. Delahanty-Fernández A, Valdivia-Álvarez I, TrujilloBrito J, Hernández-Marín M, Gómez-Cordero I, Ventura-Paz J, et al. Respuesta de anticuerpos IgM contra epítopos inmunogénicos del virus de la hepatitis A. Revista Biomédica 2004; 15: 11-6.

2. Montano A, Barañano R, Lageard B, Moratorio G, Dibarboure H, García A, et al. Prevalencia de hepatitis A en niños de 2 a 14 años y en población laboral de 18 a 49 años en Montevideo, Uruguay Rev Med Uruguay 2001; 17: 84-98.

3. Cofré J, Abarca K. Vacuna anti hepatitis A. Rev Chil Infectol 2003; 20: 243-52.

4. Bell B, Wasley A, Shapiro C, H M. Prevention of hepatitis A through active or passive immunization. Recommendations of the Advisory Committee on Immunization Practices (ACIP). Atlanta: CDC 1999.

5. Innis BL, Snitbhan R, Kunasol P, Laorakpongse T, 
Poopatanakool W, Kozik CA, et al. Protection against hepatitis A by an inactivated vaccine. JAMA 1994; 271: 1328-34.

6. Mayorga Pérez O, Herzog C, Zellmeyer M, Loaisiga A, Frosner G, Egger M. Efficacy of virosome hepatitis A vaccine in young children in Nicaragua: randomized placebo-controlled trial. J Infect Dis. 2003; 188: 671-7.

7. Fiore A, Wasley A, Bell B. Prevention of Hepatitis A Through Active or Passive Immunization. Recommendations of the Advisory Committee on Immunization Practices (ACIP). Atlanta: CDC; 2006 May 19, 2006

8. WHO. Hepatitis A Vaccine Geneva: World Health Organization 2003 [updated 2003; cited 2009]; Available from: http://www.who.int/vaccines/en/hepatitisa.shtml.

9. Taylor M, García Z, Holst Schumacher I, Somogye T, L C, Visoná K. Seroprevalencia de los virus de la hepatitis A y B en grupos etarios de Costa Rica. Act Méd Cost 2001; 43: 153-8.

10. INS. Vigilancia Rutinaria. Bogotá DC: Instituto Nacional de Salud 2009 [updated 2009; cited Feb de 2009]; Available from: http://www.ins.gov.co/?idcategoria $=1728$.

11. DANE. Proyecciones nacionales y departamentales de población. 2006-2020. Bogotá DC: Departamento Administrativo Nacional de Estadísticas 2007 Contract No: Document Number|.

12. Tapia-Conyer R, Santos JI, Cavalcanti AM, Urdaneta E, Rivera L, Manterola A, et al. Hepatitis A in Latin America: a changing epidemiologic pattern. Am J Trop Med Hyg 1999; 61: 825-9.

13. Fix AD, Martin OS, Gallicchio L, Vial PA, Lagos R. Age-specific prevalence of antibodies to hepatitis A in Santiago, Chile: risk factors and shift in age of infection among children and young adults. Am J Trop Med Hyg 2002; 66: 628-32.

14. Kleinbaum D, Kupper L, Morgenstern H. Epidemiologic Research. New York: Van Nostrand Reinhold. 1981.

15. Leske MC, Ederer F, Podgor M. Estimating incidence from age-specific prevalence in glaucoma. Am J Epidemiol 1981; 113: 606-13.

16. Fix AD, Martin OS, Gallicchio L, Vial PA, Lagos R. Age-specific prevalence of antibodies to hepatitis A in Santiago, Chile: risk factors and shift in age of infection among children and young adults. Am J Trop Med Hyg 2002; 66: 628-32.

17. Armstrong GL, Bell BP. Hepatitis A virus infections in the United States: model-based estimates and implications for childhood immunization. Pediatrics 2002; 109: 839-45.

18. Rein DB, Hicks KA, Wirth KE, Billah K, Finelli L, Fiore $\mathrm{AE}$, et al. Cost-effectiveness of routine childhood vaccination for hepatitis A in the United States. Pediatrics 2007; 119: e12-21.

19. Abarca K II, Perret C, Vial P, Zinsou JA. Immunogenicity, safety, and interchangeability of two inactivated hepatitis A vaccines in Chilean children. Vaccine 2008; 12: 270-7.

20. DANE. Defunciones no fetales 2007. Bogotá DC: Departamento Administrativo Nacional de Estadísticas 2009 [updated 2009; cited 2009 Mayo]; Available from: http://www.dane.gov.co/daneweb_V09/index. php?option $=$ com_content $\&$ view $=$ article $\&$ id $=205 \&$ Ite $\operatorname{mid}=119$.

21. Andrei Jouravlev. Drinking water supply and sanitation services on the threshold of the XXI century. Santiago, Chile: CEPAL; December 2004 [December 2004; cited 2009]; disponible en: http://www.eclac.org/publicaciones/xml/9/19539/lc12169i.pdf.

22. Tanaka J. Hepatitis A shifting epidemiology in Latin America. Vaccine 2000; 18: S57-60.

23. Ellis A, Ruttimann RW, Jacobs RJ, Meyerhoff AS, Innis BL. Cost-effectiveness of childhood hepatitis A vaccination in Argentina: a second dose is warranted. Rev Panam Salud Publica 2007; 21:345-56.

24. López E, Debbag R, Coudeville L, Baron-Papillon F, Armoni J. The cost-effectiveness of universal vaccination of children against hepatitis A in Argentina: results of a dynamic health-economic analysis. J Gastroenterol 2007; 42: $152-60$.

25. Valenzuela MT, Jacobs RJ, Arteaga O, Navarrete MS, Meyerhoff AS, Innis BL. Cost-effectiveness of universal childhood hepatitis A vaccination in Chile. Vaccine 2005; 23: 4110-9.

26. Ciocca M. Clinical course and consequences of hepatitis A infection. Vaccine 2000; 18: S71-4. 